POPULATION: This was a retrospective cohort study. We identified thirteen gynecologic oncology patients scheduled for minimally invasive surgeries (laparoscopic and robot-assisted) between March 2017 and July 2017. These patients served as the pilot for a quality improvement project comprised of a preoperative teaching session by a gynecologic oncology registered nurse (RN). Patients also received an educational booklet, designed by gynecologic oncology care team members including nurses and physicians. Educational topics included expectations for pre-, intra-, and post-operation; guidelines for post-op care at home, important contact information, and postoperative medication instructions. Patients were also given a prescription for their post-operative medications and asked to fill them before their surgeries. Following their surgeries, patients were asked to take a voluntary and anonymous 8-item online survey assessing their satisfaction with the educational intervention, their procedure, and their hospital stay. We matched these patients in a one-to-two ratio, to patients not included in the pilot, on the basis of surgery type and age. All subjects and controls spoke English as their primary language. We abstracted data from the electronic medical record including pathologic diagnosis, number of postoperative communications (telephone and email), content of postoperative communications, and various clinical characteristics. Outcome measures include length-of-stay, discharge-by-noon rate, percentage of patients with postoperative questions, and number of postoperative concerns communicated via telephone or email. RESULTS/ ANTICIPATED RESULTS: There were 39 patients in the final cohort; thirteen of whom who participated in the pilot project, POET (Perioperative Educational Tool). Thirty-nine percent of POET patients had questions regarding their surgery or post-op care as compared with $61.5 \%$ of controls $(\mathrm{P}=0.087)$. Nineteen percent of controls had questions about their postoperative medications, compared with zero percent of POET patients $(P=0.046)$. POET patients had an average of 0.69 postoperative communications, compared with 1.12 in controls. The length of stay was 8 hours in POET patients and 26 hours in controls $(\mathrm{P}=0.317)$. The discharge before noon rate was $20 \%$ in POET patients and $25 \%$ in controls $(\mathrm{P}=0.41)$. Of the 10 POET patients who completed the anonymous online survey, $100 \%$ liked the approach to teaching; $100 \%$ felt that they received consistent information regarding surgery, hospital stay, and post-op care, $100 \%$ felt prepared at discharge, $100 \%$ picked up their postoperative medications without difficulty prior to surgery. DISCUSSION/SIGNIFICANCE OF IMPACT: Patients uniformly had a positive response to their preoperative education. Although our primary outcomes were not statistically significant, the results of this unpowered, observational study suggest that anticipatory education such as we provided, may decrease the burden of postoperative communications related to surgical expectations. A preoperative teaching intervention may be especially valuable in educating patients about their postoperative medications. Although POET patients had significantly fewer questions about their postoperative medications, refilling their medications before their procedures did not seem to have an effect on discharge-by-noon rates. This may demonstrate that delays in disposition are not influenced by postoperative prescriptions. One limitation of our study is that we did not prospectively measure patient satisfaction with surgical care. Another limitation is that the pilot educational intervention was conducted entirely in English. To our knowledge, there exists no analysis of the effect of English language proficiency on outcomes such as patient satisfaction, length of stay, and discharge-by-noon rates, and other clinical outcomes in this surgical patient population. It is well-demonstrated in the literature that limited English proficiency contributes negatively to health care quality. Our next steps involve establishing a prospective study to measure the effects of preoperative education on patient satisfaction with their procedure, post-operative communications, and discharge by noon rates. We also plan to administer POET to Spanish- and Chinese-language speakers, to better understand the effect of limited English proficiency on our outcome measures of interest.

3423

\section{Poorer Outcomes Among Septic Patients with Protein Energy Malnutrition}

Adeyinka Charles Adejumo ${ }^{1}$, Olalekan Akanbi and Lydie Pani ${ }^{1}$ North Shore Medical Center

OBJECTIVES/SPECIFIC AIMS: Protein Energy Malnutrition (PEM) could compromise the body's defense systems resulting in sepsis, which further depletes calorie stores. Among hospitalized patients, we investigate 1) the relationship between PEM and sepsis, 2) the impact of PEM on trends in mortality from sepsis, and 3) the influence of PEM on clinical outcomes of sepsis. METHODS/STUDY POPULATION: Using the 2014 Healthcare Cost and Utilization Project - Nationwide Inpatient Sample (NIS) patient's discharge records, we identified patients with sepsis, PEM, and other clinical conditions with ICD-9-CM codes. After stratifying sepsis into two: uncomplicated (without shock) and complicated (with shock), we estimated the adjusted odds (aOR) of developing sepsis (total, uncomplicated and complicated) with PEM. Then, we selected hospitalizations with sepsis from 2007-2014 years of the HCUP-NIS, and calculated the trend in mortality from sepsis, stratified by PEM status, as an effect modifier. Finally, we matched PEM to no PEM (1:1) using a greedy algorithm-based propensity methodology and estimated the effect of having mortality, complicated sepsis and 10 other clinical outcomes and healthcare utilization (SAS 9.4). RESULTS/ANTICIPATED RESULTS: PEM was associated with higher odds for sepsis (aOR:3.97[3.89-4.05]), and complicated vs. uncomplicated sepsis (1.74[1.67-1.81]). Although mortality in sepsis has been trending down from 2007-2014 (-1.19\%/year, p-trend $<0.0001)$, the decrease was less pronounced among those with PEM vs. no-PEM ( $-0.86 \% /$ year vs. $-1.29 \%$ /year, p-value $<0.0001)$. After propensity matching, PEM was associated with higher mortality (1.35[1.32-1.37]), cost $(\$ 160,724[159,517-161,940]$ vs. $\$ 86,650[85,931-87,375])$, length of stay $(14.76[14.68-14.84]$ vs. $8.49[8.45-8.56]$ days), and worse outcomes in general. DISCUSSION/ SIGNIFICANCE OF IMPACT: PEM is a risk factor of sepsis and associated with poorer outcomes among septic patients. A concerted effort involving primary care physicians, nutritionists, nurses in identifying, preventing, and treatment of PEM in the communitydwelling individuals before hospitalization might mitigate against these devastating outcomes.

3435

\section{Predicting Linkage-to-Care Outcomes Among Patients Screened for Hepatitis $C$ in an Urban Academic Emergency Department}

Joshua Blackwell ${ }^{1}$, Joel Rodgers and Erik Hess

${ }^{1}$ University of Alabama at Birmingham

OBJECTIVES/SPECIFIC AIMS: The purpose of the current study is to determine predictors of linkage-to-care (LTC) outcomes among patients screened for hepatitis $\mathrm{C}$ virus (HCV) infection in the emergency department (ED). The study is one of the first to report the 
impact of factors influencing medical eligibility for HCV treatment. By establishing predictors of linkage outcomes, we hope to characterize patients at highest risk of being lost to follow-up. This information can be used to guide the development of targeted interventions and optimize linkage efforts at institutions with similar programs. METHODS/STUDY POPULATION: In 2013, we implemented an opt-out HCV screening program targeting baby boomers presenting to the ED. The screening program was expanded in 2015, and all patients who presented to the ED (an academic ED with 73,000 annual visits) between 2015 and 2018 were offered an HCV antibody test. Confirmatory RNA tests were ordered for all patients with a positive screening test, and those with confirmed infection were contacted (when possible) by a linkage coordinator, who sought to facilitate linkage to outpatient care. Patients were followed until they had been successfully linked to care or were lost to follow-up; successful linkage was defined as a patient attending a scheduled HCV clinic visit. Linkage outcomes (linked vs. not linked) among confirmed cases were analyzed based on previously described LTC barriers, sociodemographic factors, and factors influencing $\mathrm{HCV}$ treatment eligibility. Binary multiple logistic regression was used to model the relationship between predictors and LTC outcomes. RESULTS/ANTICIPATED RESULTS: Between August 2015 and June 2018, 48,947 patients were screened for HCV, yielding 4,026 positive results. HCV infection was confirmed in 1,798 patients, 1,651 of whom were eligible for the current study. By July 2018, 884 eligible patients $(53.5 \%)$ had been successfully linked to care. Odds of linkage failure were decreased among patients with known linkage barriers including mental health disorders (adjusted odds ratio $[\mathrm{aOR}], 0.28 ; 95 \%$ confidence interval [CI], 0.21-0.37), active substance abuse (aOR, 0.65; 95\% CI, 0.47-0.91), and 'competing' medical comorbidities (aOR, 0.31; 95\% CI, 0.21-0.47); however, odds of linkage failure were increased in the presence of medical comorbidities leading to ineligibility for HCV treatment (aOR, 21.71; 95\% CI, 7.02-67.13). Decreased odds of linkage failure were also found among patients belonging to the baby boomer population (aOR, 0.64; 95\% CI, 0.46-0.91) and individuals with HBV co-infection (aOR, 0.36; 95\% CI, 0.14-0.94). Finally, odds of linkage failure were increased among patients without health insurance (aOR, 1.952; 95\% $\mathrm{CI}, 1.01-3.79$ ) and with public health insurance (aOR, 2.140; 95\% CI, 1.08-4.24). While African-American patients exhibited decreased odds of linkage failure in bivariate analyses, this association was not significant in the multivariate analysis. Variables ultimately found not to be predictive of linkage outcome included race, ethnicity, gender, homelessness, and incarceration. DISCUSSION/ SIGNIFICANCE OF IMPACT: In contrast with current trends in the literature, our analysis found that patients with any of three well-described linkage barriers (mental health disorder, active substance abuse, and 'competing' comorbidities) exhibited decreased odds of linkage failure. It is possible that knowledge of these barriers by linkage personnel led to increased persistence of efforts for such patients, resulting in improved linkage among patients with these barriers. However, another possibility is that individuals with factors such as a mental health disorder or medical comorbidity were more likely to experience a clinical encounter, during which discussions about HCV treatment could take place. It is also possible that other contributing factors were unaccounted for by our analysis, such as internal programmatic issues, patient education and income levels, marital status, or other 'destabilizing' factors that would reduce a patient's likelihood of being contacted by a linkage coordinator. While patients with general medical comorbidities were less likely to experience linkage failure, patients who were ineligible for $\mathrm{HCV}$ treatment due to a medical comorbidity were significantly more likely to experience linkage failure. While this finding is intuitive, it is nonetheless troubling, as we would expect patients with severe medical comorbidities to benefit greatly from health counseling, mental health services, substance abuse treatment, or other resources available through linkage. As a result of this finding, we intend to adapt our approach to medically ineligible patients, emphasizing the availability and potential usefulness of such resources. It is unsurprising that uninsured and publicly insured patients were less likely to be successfully linked to care, as HCV treatment continues to be associated with significant financial costs for such patients. However, it is also possible that confounding factors such as education and income level, which were not included in our analysis, would be more predictive of patients' ability or willingness to undergo HCV treatment. More research is needed to improve linkage-to-care for patients with HCV. In the near future, we intend to conduct an internal evaluation, as well as a positive deviance analysis of other LTC programs, to determine additional ways to improve linkage outcomes for all patients screened for HCV in the ED.

\section{7}

Quantifying the psychosocial benefits of masculinizing chest reconstruction in transgender men with patient reported outcomes: The GENDER-Q

Adrienne Kennedy ${ }^{1}$, Andre Alcon and Esther Kim

${ }^{1}$ University Of California, San Francisco

OBJECTIVES/SPECIFIC AIMS: 1 . To evaluate the effects of masculinizing chest reconstruction on the quality of life of female-to-male transgender individuals. 2. To develop and validate a quality of life survey for female-to-male transgender individuals undergoing masculinizing chest reconstruction gender-affirming surgery. METHODS/ STUDY POPULATION:. We developed and refined the GENDER-Q using focus groups and exploratory interviews with FTM individuals that were recorded, transcribed, and coded. All consenting FTM patients undergoing chest reconstruction at UCSF Parnassus Medical center between 2017-2019 who meet World Professional Association for Transgender Health (WPATH) criteria for gender dysphoria will be enrolled in the study. The GENDER-Q will be co-administered with the WHO Quality of Life-BREF survey pre-operatively, 6 weeks post-operatively, and one year post-operatively through REDCAP, an online survey database. RESULTS/ANTICIPATED RESULTS: Approximately 70 patients will be enrolled in the study. Preliminary results detect statistically significant mean quality of life postoperative improvements in all three sections (Physical Health, Gender Presentation, Psychological Health) of the GENDER-Q at 6 -week follow up that is maintained at one-year $(p<0.005)$. Statistically significant improvements were similarly achieved and maintained with the WHO QOL-BREF survey $(\mathrm{p}<0.05)$. The mean reported improvement from baseline to follow-up appears to be more pronounced in the GENDER-Q survey compared to WHO QOL-BREF. The median time to complete the pre and post-operative surveys was 10 minutes. Calculation of Cronbach's (0.67-0.81) and the Pearson Correlation Coefficient for each section revealed excellent internal validity. DISCUSSION/SIGNIFICANCE OF IMPACT: There are few studies assessing quality of life outcomes in transgender patients undergoing gender-affirming surgeries. A standardized and validated assessment tool will provide the means for which data can be pooled in large multi-center studies. Providing further evidence to support the positive health outcomes of gender-affirming surgeries can lead to broader access and reduce healthcare disparities among transgender populations. 